\title{
ON A STOCHASTIC HYPERBOLIC SYSTEM IN LINEAR ELASTICITY*
}

\author{
JONG UHN $\mathrm{KIM}^{\dagger}$
}

\begin{abstract}
In this paper we discuss the Cauchy problem for linear elasticity with a space-time white noise forcing term. We show that the solution can be represented by a formula analogous to the Riesz formula for solutions of a wave equation. The solution is a generalized stochastic process and is obtained as the limit of a sequence of ordinary stochastic processes. Our basic tool is the Hilbert space method combined with geometric properties of solutions inherent with a hyperbolic system.
\end{abstract}

Key words. generalized stochastic process, Gaussian process, white noise, hyperbolic system

AMS subject classifications. 35L15, 35R60, 60H15

PII. S0036141099350377

0. Introduction. In this paper we present new results on the Cauchy problem for a hyperbolic system with a white noise forcing term associated with linear elasticity:

$$
\begin{gathered}
u_{t t}(t, x)=A\left(t, x, D_{x}\right) u(t, x)+F(t, x) \xi(t, x) \quad \text { in }(0, \infty) \times R^{n} ; \\
u(0, x)=0, \quad u_{t}(0, x)=0, \quad \text { in } R^{n},
\end{gathered}
$$

where $x=\left(x_{1}, \ldots, x_{n}\right) \in R^{n}, u=\left(u_{1}, \ldots, u_{n}\right)$ denotes the displacement from equilibrium. $A\left(t, x, D_{x}\right)$ is a second order matrix differential operator, $F(t, x)$ is a matrix function, and $\xi(t, x)$ stands for a vector-valued space-time white noise. Here we only focus on the nonhomogeneous forcing term, and assume the zero initial conditions. Since the system is linear, nonzero initial conditions can be handled separately. The motion of a one-dimensional elastic medium driven by a random noise is typically described by a one-dimensional wave equation with a space-time white noise. This was discussed by Walsh [15] in the framework of Brownian sheets. A numerical result is shown on the front cover of the monograph [7]. Other versions were investigated by Cabaña [1] and Orsingher [13]. It was also discussed by Da Prato and Zabczyk [3] as an example of abstract evolution equations. A semilinear hyperbolic equation with onedimensional space variable was discussed by Nualart [12] as a two-parameter stochastic differential equation. Marcus and Mizel [10] studied initial-boundary value problems for a stochastic hyperbolic system in one space dimension. A two-dimensional semilinear wave equation was discussed by Dalang and Frangos [4], and Mueller [11] when the random noise is a white noise in time with smooth spatial covariance.

The purpose of this work is to establish the existence of a solution of (0.1) and (0.2) by a representation formula analogous to the Riesz formula for solutions of a wave equation. The representation formula gives good information on the structure of the solution which is a generalized stochastic process. This motivates our quest for the representation formula. Riesz [14] used analytical theory of integrals of fractional

\footnotetext{
* Received by the editors January 4, 1999; accepted for publication (in revised form) January 24, 2000; published electronically July 5, 2000.

http://www.siam.org/journals/sima/32-2/35037.html

${ }^{\dagger}$ Department of Mathematics, Virginia Tech, Blacksburg, VA 24061-0123 (kim@math.vt.edu).
} 
order to establish the formula

$$
u(t, x)=\frac{1}{2^{n} \pi^{(n-1) / 2}((n-1) / 2) !} \Lambda^{(n-1) / 2} \int_{\Xi(t, x)} f(s, y) d y d s,
$$

where $n \geq 3$ is an odd integer, $x \in R^{n}, t \geq 0, \Xi(t, x)$ is the backward light cone defined by $(t-s)^{2} \geq|x-y|^{2}, \quad 0 \leq s \leq t$. Here, $u$ is the solution of

$$
\begin{gathered}
\Lambda u=f \quad \text { for } x \in R^{n}, t \geq 0 ; \\
u(0, x)=0, u_{t}(0, x)=0 \quad \text { for } x \in R^{n},
\end{gathered}
$$

where $\Lambda=\partial_{t t}-\Delta$, and $\Delta$ is the Laplacian.

Gaveau [6] applied this formula to the Cauchy problem for a three-dimensional wave equation with a space-time white noise. For a one-dimensional wave equation, the solution can be represented by a stochastic integral because the integral kernel is locally $L^{2}$, which is not true in the higher-dimensional case. Gaveau overcame this difficulty by means of the above Riesz formula, for the above integral in (0.3) can be well-defined as a stochastic integral when $f d y d s$ is replaced by $f \xi(d y d s)$ where $f$ is locally bounded and $\xi$ is the white noise. Thanks to the explicit structure of the formula, he could first construct a continuous martingale (according to a partial ordering defined in terms of the backward light cone) through the Riesz integral, and then, a solution was obtained by applying the wave operator to this continuous stochastic process. Hence the resulting solution is a generalized stochastic process. However, the particular formula used in [6], which easily generates a martingale, is valid only for odd space dimensions. He also showed that the Cauchy value of this generalized stochastic process is well-defined by the formula of integration by parts.

This work is an outgrowth of our effort to obtain a similar representation formula for the system of equations in linear elasticity. We will show that the unique solution $u$ of the above Cauchy problem (0.1)-(0.2) in any space dimension can be obtained as a generalized stochastic process in the following form:

$$
u(t, x ; \omega)=L^{d} V(t, x ; \omega),
$$

where $L=\partial_{t t} I-A\left(t, x, D_{x}\right), I$ is the $n \times n$ identity matrix, $d$ is the smallest integer larger than $(n / 2)-1$, and $V$ is a continuous Gaussian process which satisfies the property of a domain of dependence. In particular, when $n \geq 3$ is an odd integer, $d=(n-1) / 2$. Since the Riesz formula is not available in our case, we have to employ an entirely different argument. The core task is to obtain an integral kernel. For this, we use the Hilbert space method combined with the property of a domain of dependence. We then approximate the space-time white noise by truncating the chaos expansion. The corresponding approximate solution is an ordinary stochastic process, and the true solution is obtained as the limit. Our results are completely new and our approach is different from those of all the previous works.

1. Preliminaries and statement of the main result. Throughout this paper, we make the following assumptions.

The matrix operator $A\left(t, x, D_{x}\right)$ is given by

$$
A_{i j}\left(t, x, D_{x}\right)=\sum_{k, l=1}^{n} C_{i j}^{k l}(t, x) \frac{\partial^{2}}{\partial x_{k} \partial x_{l}}+\sum_{k=1}^{n} D_{i j}^{k}(t, x) \frac{\partial}{\partial x_{k}}
$$


for $i, j=1, \ldots, n$. All coefficients are real valued and $R^{n+1}$;

(I) $C_{i j}^{k l}(t, x) \in C^{\infty}\left(R^{n+1}\right)$ and all the derivatives of each $C_{i j}^{k l}$ are bounded on

(II) $C_{i j}^{k l}(t, x)=C_{k j}^{i l}(t, x)=C_{j i}^{l k}(t, x)$ for all $(t, x) \in R^{n+1}$ and every $i, j, k$, and $l$;

(III) there is a positive constant $c_{0}$ such that

$$
\sum_{k, l, i, j} C_{i j}^{k l}(t, x) \epsilon_{k i} \epsilon_{l j} \geq c_{0} \sum_{k, i} \epsilon_{k i} \epsilon_{k i}
$$

for all $(t, x) \in R^{n+1}$ and every symmetric tensor $\epsilon_{k i}$; $R^{n+1}$;

(IV) $D_{i j}^{k}(t, x) \in C^{\infty}\left(R^{n+1}\right)$ and all the derivatives of each $D_{i j}^{k}$ are bounded on

(V) $F(t, x)$ is an $n \times n$ matrix function whose components are all measurable and bounded on each bounded subset of $R^{n+1}$.

We now list some known facts about the following deterministic Cauchy problem:

$$
\begin{gathered}
u_{t t}(t, x)=A\left(t, x, D_{x}\right) u(t, x)+f(t, x) \quad \text { in }(0, \infty) \times R^{n} ; \\
u(0, x)=u_{0}(x), \quad u_{t}(0, x)=u_{1}(x) \quad \text { in } R^{n} .
\end{gathered}
$$

Let a real number $s$ and a positive number $T$ be given.

TheOREM 1.1. For given $f(t, x) \in L^{2}\left(0, T ;\left(H^{s}\left(R^{n}\right)\right)^{n}\right), u_{0}(x) \in\left(H^{s+1}\left(R^{n}\right)\right)^{n}$, and $u_{1}(x) \in\left(H^{s}\left(R^{n}\right)\right)^{n}$, there is a unique solution $u(t, x)$ of (1.2) and (1.3) in $C\left([0, T] ;\left(H^{s+1}\left(R^{n}\right)\right)^{n}\right) \cap C^{1}\left([0, T] ;\left(H^{s}\left(R^{n}\right)\right)^{n}\right)$.

Here $H^{s}\left(R^{n}\right)$ denotes the usual Sobolev space. For the technical details of the proof, see [9].

Next we fix any $t_{0} \geq 0$ and $x_{0} \in R^{n}$ and define for $0 \leq t \leq t_{0}, \epsilon>0$ and $\eta>0$,

$$
\Gamma_{\left(t_{0}, x_{0}\right)}(t ; \epsilon, \eta)=\left\{x \in R^{n}|| x-x_{0} \mid<\epsilon+\eta\left(t_{0}-t\right)\right\} .
$$

$\Gamma_{\left(t_{0}, x_{0}\right)}(t ; \epsilon, \eta)$ is an $n$-dimensional ball for each $0 \leq t \leq t_{0}$, and $\cup_{0 \leq t \leq t_{0}}\left\{\left(t, \overline{\Gamma_{\left(t_{0}, x_{0}\right)}(t ; \epsilon, \eta)}\right)\right\}$ is a truncated cone in $R^{n+1}$.

ThEOREM 1.2. Let $s=m$ be a nonnegative integer, and $T>0$ be given. Choose any $\left(t_{0}, x_{0}\right) \in[0, T] \times R^{n}$. Then there is $\eta>0$ depending on the coefficients of $A\left(t, x, D_{x}\right)$, but independent of $u, f,\left(t_{0}, x_{0}\right)$, and $\epsilon$ such that the above solution satisfies

$$
\begin{gathered}
\left\|u\left(t_{0}, \cdot\right)\right\|_{\left(H^{m+1}\left(\Gamma\left(t_{0} ; \epsilon, \eta\right)\right)\right)^{n}}^{2}+\left\|u_{t}\left(t_{0}, \cdot\right)\right\|_{\left(H^{m}\left(\Gamma\left(t_{0} ; \epsilon, \eta\right)\right)\right)^{n}}^{2} \\
\leq M\left(\left\|u_{0}\right\|_{\left(H^{m+1}(\Gamma(0 ; \epsilon, \eta))\right)^{n}}^{2}+\left\|u_{1}\right\|_{\left(H^{m}(\Gamma(0 ; \epsilon, \eta))\right)^{n}}^{2}+\int_{0}^{t_{0}}\|f(h, \cdot)\|_{\left(H^{m}(\Gamma(h ; \epsilon, \eta))\right)^{n}}^{2} d h\right),
\end{gathered}
$$

where $M$ is a positive constant independent of $u, f,\left(t_{0}, x_{0}\right)$, and $\epsilon$. Here the subscript $\left(t_{0}, x_{0}\right)$ of $\Gamma$ has been suppressed.

This has been established for a general first order hyperbolic system in [2]. The same argument can be applied to a second order hyperbolic system. See [5] and [9].

COROLlary 1.3. Suppose that the support of $u_{0}$ and $u_{1}$ is disjoint from $\overline{\Gamma_{\left(t_{0}, x_{0}\right)}(0 ; \epsilon, \eta)}$, and the support of $f$ is disjoint from $\cup_{0 \leq t \leq t_{0}}\left\{\left(t, \overline{\Gamma_{\left(t_{0}, x_{0}\right)}(t ; \epsilon, \eta)}\right)\right\}$ for some $\left(t_{0}, x_{0}\right) \in[0, \infty) \times R^{n}$ in Theorem 1.1. Then the support of $u$ is disjoint from $\cup_{0 \leq t \leq t_{0}}\left\{\left(t, \overline{\Gamma_{\left(t_{0}, x_{0}\right)}(t ; \epsilon, \eta)}\right)\right\}$. 
If $s<0$, this can be proved by approximating the solution by a sequence of solutions with smooth initial data and $f$, for which (1.5) can be applied.

Next we set up a base probability space for generalized stochastic processes. Let $\mathcal{S}=\left(\mathcal{S}\left(R^{n+1}\right)\right)^{n}$ be the space of $R^{n}$-valued rapidly decreasing $C^{\infty}$ functions on $R^{n+1}$, and $\mathcal{S}^{\prime}=\left(\mathcal{S}^{\prime}\left(R^{n+1}\right)\right)^{n}$ be its dual equipped with the weak-star topology. $\mathcal{B}\left(\mathcal{S}^{\prime}\right)$ stands for the set of all Borel subsets of $\mathcal{S}^{\prime}$. By the Bochner-Minlos theorem, there is a probability measure $\mu$ on $\mathcal{B}\left(\mathcal{S}^{\prime}\right)$ such that

$$
\int_{\mathcal{S}^{\prime}} \exp \left(\sqrt{-1}\langle\omega, \phi\rangle_{n}\right) d \mu(\omega)=\exp \left(-\|\phi\|_{\left(L^{2}\left(R^{n+1}\right)\right)^{n}}^{2} / 2\right)
$$

for all $\phi \in \mathcal{S}$. Here, $\langle\cdot, \cdot\rangle_{n}$ denotes the duality pairing between $\mathcal{S}^{\prime}$ and $\mathcal{S}$, i.e.,

$$
\langle\omega, \phi\rangle_{n}=\sum_{i=1}^{n}\left\langle\omega_{i}, \phi_{i}\right\rangle
$$

for $\omega=\left(\omega_{1}, \ldots, \omega_{n}\right), \phi=\left(\phi_{1}, \ldots, \phi_{n}\right)$, where $\langle\cdot, \cdot\rangle$ denotes the duality pairing between $\mathcal{S}^{\prime}\left(R^{n+1}\right)$ and $\mathcal{S}\left(R^{n+1}\right)$. By (1.6), it follows that for each $f \in\left(L^{2}\left(R^{n+1}\right)\right)^{n}$, $\langle\omega, f\rangle_{n}$ can be defined to be a random variable on the probability space $\left(\mathcal{S}^{\prime}, \mathcal{B}\left(\mathcal{S}^{\prime}\right), \mu\right)$, and, for each positive integer $k$,

$$
E\left(\left|\langle\omega, f\rangle_{n}\right|^{2 k}\right)=\int_{\mathcal{S}^{\prime}}\left|\langle\omega, f\rangle_{n}\right|^{2 k} d \mu=\frac{(2 k) !}{k ! 2^{k}}\|f\|_{\left(L^{2}\left(R^{n+1}\right)\right)^{n}}^{2 k} .
$$

For more details, see [7]. The above white noise $\xi(t, x)$ is a vector-valued generalized stochastic process in the sense that

$$
\xi_{\phi}(\omega)=\left(\left\langle\omega_{1}, \phi_{1}\right\rangle, \ldots,\left\langle\omega_{n}, \phi_{n}\right\rangle\right)
$$

is a vector-valued random variable on $\left(\mathcal{S}^{\prime}, \mathcal{B}\left(\mathcal{S}^{\prime}\right), \mu\right)$ for each $\phi \in\left(C_{0}^{\infty}\left(R^{n+1}\right)\right)^{n}$. In the meantime, $F \xi$ is a vector-valued generalized stochastic process defined by

$$
(F \xi)_{\phi}(\omega)=\left(\sum_{j=1}^{n}\left\langle\omega_{j}, F_{1 j} \phi_{1}\right\rangle, \ldots, \sum_{j=1}^{n}\left\langle\omega_{j}, F_{n j} \phi_{n}\right\rangle\right),
$$

for each $\phi \in\left(C_{0}^{\infty}\left(R^{n+1}\right)\right)^{n}$. Next we define for each $i, j=1, \ldots, n$,

$$
B_{i j}\left(x_{0}, x_{1}, \ldots, x_{n} ; \omega\right)=\left\langle\omega_{j}(y), F_{i j}(y) \chi_{\left(x_{0}, \ldots, x_{n}\right)}(y)\right\rangle .
$$

Here, $y \in R^{n+1}$ denotes the variable for the duality action $\langle\cdot, \cdot\rangle$, and

$$
\chi_{\left(x_{0}, \ldots, x_{n}\right)}\left(y_{0}, \ldots, y_{n}\right)=\left\{\begin{array}{c}
(-1)^{k} \quad \begin{array}{r}
\text { if } 0 \leq y_{i}<x_{i} \text { or } x_{i}<y_{i}<0 \\
\text { for each } i=0,1, \ldots, n,
\end{array} \\
\text { otherwise }
\end{array}\right.
$$

where $k$ is the number of negative $x_{i}$ 's. For each bounded subset $K$ of $R^{n+1}$, it holds that

$$
\int_{R^{n+1}}\left|\chi_{\left(x_{0}, \ldots, x_{n}\right)}(y)-\chi_{\left(\tilde{x}_{0}, \ldots, \tilde{x}_{n}\right)}(y)\right|^{2} d y \leq C_{K}\left|\left(x_{0}, \ldots, x_{n}\right)-\left(\tilde{x}_{0}, \ldots, \tilde{x}_{n}\right)\right|
$$


for all $\left(x_{0}, \ldots, x_{n}\right),\left(\tilde{x}_{0}, \ldots, \tilde{x}_{n}\right) \in K$, for some positive constant $C_{K}$. Thus it follows from (1.7) that for each bounded subset $K$ of $R^{n+1}$ and each positive integer $m$,

$$
\begin{aligned}
& E\left(\left|B_{i j}\left(x_{0}, \ldots, x_{n} ; \omega\right)-B_{i j}\left(\tilde{x}_{0}, \ldots, \tilde{x}_{n} ; \omega\right)\right|^{2 m}\right) \\
& \leq C_{m, K}\left|\left(x_{0}, \ldots, x_{n}\right)-\left(\tilde{x}_{0}, \ldots, \tilde{x}_{n}\right)\right|^{m}
\end{aligned}
$$

for all $\left(x_{0}, \ldots, x_{n}\right),\left(\tilde{x}_{0}, \ldots, \tilde{x}_{n}\right) \in K$, for some positive constant $C_{m, K}$. By a partition of unity and the Kolmogorov continuity theorem, there is a continuous version of $B_{i j}\left(x_{0}, \ldots, x_{n} ; \omega\right)$. See [8] and [12] for the multiparameter version of Kolmogorov's theorem. From now on, we always mean this continuous version.

Lemma 1.4. For each $\phi=\left(\phi_{1}, \ldots, \phi_{n}\right)$ with $\phi_{i} \in C_{0}^{\infty}\left(R^{n+1}\right), i=1, \ldots, n$, it holds that

$$
\begin{aligned}
& \text { the ith component of }(F \xi)_{\phi}(\omega) \\
& =\sum_{j=1}^{n} \int_{R^{n+1}}(-1)^{n+1} \frac{\partial^{n+1} \phi_{i}}{\partial x_{0} \cdots \partial x_{n}} B_{i j}\left(x_{0}, \ldots, x_{n} ; \omega\right) d x_{0} \ldots d x_{n}
\end{aligned}
$$

for almost all $\omega$.

Proof. Since $B_{i j}\left(x_{0}, \ldots, x_{n} ; \omega\right)$ is a continuous version of the process defined by (1.10), $B_{i j}\left(x_{0}, \ldots, x_{n} ; \omega\right)$ is continuous in $\left(x_{0}, \ldots, x_{n}\right)$, for each $\omega$, and there is a subset $\Omega \in \mathcal{B}\left(\mathcal{S}^{\prime}\right)$ with $\mu(\Omega)=1$, such that for each $\omega \in \Omega$,

$$
B_{i j}\left(r_{0}, \ldots, r_{n} ; \omega\right)=\left\langle\omega_{j}(y), F_{i j}(y) \chi_{\left(r_{0}, \ldots, r_{n}\right)}(y)\right\rangle
$$

for all $\left(r_{0}, \ldots, r_{n}\right)$ with $r_{i}=$ a rational number, $i=0, \ldots, n$.

Choose any $\phi \in\left(C_{0}^{\infty}\left(R^{n+1}\right)\right)^{n}$ and set

$$
\psi=\frac{\partial^{n+1} \phi}{\partial x_{0} \ldots \partial x_{n}}\left(x_{0}, \ldots, x_{n}\right) .
$$

Let $\Pi$ be a cube in $R^{n+1}$ with side length $q$ such that supp $\phi \subset \Pi$. For each $N$, we divide this cube into $N^{n+1}$ cubes of equal size. For $\nu=1, \ldots, N^{n+1}$, let $z^{\nu}$ denote an interior point of the $\nu$ th cube whose coordinates are rational numbers. Then we have, for every $\omega \in \Omega$,

$$
\begin{aligned}
\int_{R^{n+1}} \psi_{i}\left(x_{0}, \ldots, x_{n}\right) B_{i j}\left(x_{0}, \ldots, x_{n} ; \omega\right) d x_{0} \cdots d x_{n} \\
=\lim _{N \rightarrow \infty} \frac{q^{n+1}}{N^{n+1}} \sum_{\nu=1}^{N^{n+1}}\left\langle\omega_{j}(y), \quad F_{i j}(y) \psi_{i}\left(z^{\nu}\right) \chi_{z^{\nu}}(y)\right\rangle .
\end{aligned}
$$

But it is easy to see that as a function of $y=\left(y_{0}, \ldots, y_{n}\right)$,

$$
\frac{q^{n+1}}{N^{n+1}} \sum_{\nu=1}^{N^{n+1}} \psi_{i}\left(z^{\nu}\right) \chi_{z^{\nu}}(y)
$$

is bounded and compactly supported uniformly in $N$, and converges to

$$
(-1)^{k} \int_{y_{0}} \ldots \int_{y_{n}} \psi_{i}\left(x_{0}, \ldots, x_{n}\right) d x_{0} \ldots d x_{n}
$$


as $N \rightarrow \infty$, for each $y$, where $k$ is the number of negative $y_{i}$ 's and

$$
\int_{y_{i}}=\int_{y_{i}}^{\infty} \quad \text { for } y_{i} \geq 0
$$

and

$$
\int_{y_{i}}=\int_{-\infty}^{y_{i}} \quad \text { for } y_{i}<0
$$

Hence, as $N \rightarrow \infty$,

$$
\frac{q^{n+1}}{N^{n+1}} \sum_{\nu=1}^{N^{n+1}} F_{i j}(y) \psi_{i}\left(z^{\nu}\right) \chi_{z^{\nu}}(y) \rightarrow(-1)^{n+1} F_{i j}(y) \phi_{i}(y) \text { in } L^{2}\left(R_{y}^{n+1}\right) .
$$

By virtue of (1.7), (1.17), and (1.18), we have (1.14) for almost all $\omega$.

Next we consider a chaos expansion of the white noise. We follow the construction of an orthonormal basis for $L^{2}\left(R^{n+1}\right)$ in [7]. Let $\xi_{m}(t)$ be the Hermite function of $t \in R$ for $m=1,2, \ldots$ Let $\delta^{j}=\left(\delta_{0}^{j}, \delta_{1}^{j}, \ldots, \delta_{n}^{j}\right)$ be the $j$ th multi-index number in some fixed ordering of all $(n+1)$-dimensional multi-indices $\delta=\left(\delta_{0}, \ldots, \delta_{n}\right)$, each $\delta_{i}=$ a positive integer. This ordering satisfies the property

$$
i<j \quad \text { implies } \delta_{0}^{i}+\cdots+\delta_{n}^{i} \leq \delta_{0}^{j}+\cdots+\delta_{n}^{j} .
$$

We write

$$
\eta_{j}\left(x_{0}, \ldots, x_{n}\right)=\xi_{\delta_{0}^{j}}\left(x_{0}\right) \xi_{\delta_{1}^{j}}\left(x_{1}\right) \cdots \xi_{\delta_{n}^{j}}\left(x_{n}\right) .
$$

Then $\left\{\eta_{j}\right\}_{j=1}^{\infty}$ forms an orthonormal basis for $L^{2}\left(R^{n+1}\right)$. It follows that for each $\phi \in\left(C_{0}^{\infty}\left(R^{n+1}\right)\right)^{n}$,

$$
\begin{aligned}
& \text { the } i \text { th component of }(F \xi)_{\phi}(\omega)=\sum_{j=1}^{n}\left\langle\omega_{j}(y), \quad F_{i j}(y) \phi_{i}(y)\right\rangle \\
& =\lim _{N \rightarrow \infty} \sum_{k=1}^{N} \sum_{j=1}^{n}\left\langle\eta_{k}(y), F_{i j}(y) \phi_{i}(y)\right\rangle_{L^{2}\left(R_{y}^{n+1}\right)}\left\langle\omega_{j}(y), \eta_{k}(y)\right\rangle
\end{aligned}
$$

in $L^{2}\left(\mathcal{S}^{\prime}, d \mu\right)$. This will be used to construct a solution in the next section.

We adopt the following definition of a solution to (0.1).

DeFinition 1.5. A vector-valued generalized stochastic process $u\left(t, x_{1}, \ldots, x_{n} ; \omega\right)$ is a solution of $(0.1)$ in $R_{+}^{n+1}$ if there is a subset $\Omega \in \mathcal{B}\left(\mathcal{S}^{\prime}\right)$ with $\mu(\Omega)=1$ such that

$$
\ll u, \quad L^{*} \phi \gg=\sum_{i, j=1}^{n} \int_{R_{+}^{n+1}}(-1)^{n+1} \frac{\partial^{n+1} \phi_{i}}{\partial t \cdots \partial x_{n}} B_{i j}\left(t, x_{1}, \ldots, x_{n} ; \omega\right) d t \cdots d x_{n}
$$

holds for all $\phi \in\left(C_{0}^{\infty}\left(R_{+}^{n+1}\right)\right)^{n}$, for each $\omega \in \Omega$.

Here, $R_{+}^{n+1}=\left\{(t, x) \mid t>0, x \in R^{n}\right\}, \ll \cdot, \cdot \gg$ is the duality pairing of $\left(\mathcal{D}^{\prime}\left(R_{+}^{n+1}\right)\right)^{n}$ and $\left(C_{0}^{\infty}\left(R_{+}^{n+1}\right)\right)^{n}$, and $L^{*}$ is the adjoint of

$$
L=\partial_{t t} I-A\left(t, x, D_{x}\right) .
$$


Lemma 1.6. Suppose $u\left(t, x_{1}, \ldots, x_{n} ; \omega\right)$ is a solution of $(0.1)$ in $R_{+}^{n+1}$ according to the above definition, i.e., there is a subset $\Omega \in \mathcal{B}\left(\mathcal{S}^{\prime}\right)$ with $\mu(\Omega)=1$ such that for each $\omega \in \Omega$, (1.21) holds for all $\phi \in\left(C_{0}^{\infty}\left(R_{+}^{n+1}\right)\right)^{n}$. Then, for each $\omega \in \Omega, T>0$ and bounded open subset $\Delta \subset R^{n}$, we have

$$
\begin{gathered}
\psi(x) u(t, x ; \omega) \in\left(C\left([0, T] ; H^{-m+1}\left(R^{n}\right)\right)\right)^{n}, \\
\psi(x) u_{t}(t, x ; \omega) \in\left(C\left([0, T] ; H^{-m}\left(R^{n}\right)\right)\right)^{n}
\end{gathered}
$$

for all $\psi \in C_{0}^{\infty}(\Delta)$, for some positive integer $m$.

Proof. Let us set, for $i=1, \ldots, n$,

$$
\begin{aligned}
& v_{i}\left(t, x_{1}, \ldots, x_{n} ; \omega\right) \\
& =u_{i}\left(t, x_{1}, \ldots, x_{n} ; \omega\right)-\sum_{j=1}^{n} \frac{\partial^{n}}{\partial x_{1} \ldots \partial x_{n}} \int_{0}^{t} B_{i j}\left(s, x_{1}, \ldots, x_{n} ; \omega\right) d s .
\end{aligned}
$$

We fix $\omega \in \Omega, T>0$, and $\Delta \subset R^{n}$. Let $\eta>0$ be the number in Theorem 1.2. We note that Theorem 1.2 and Corollary 1.3 are also valid with respect to the reversed time variable. Choose $q>0$ such that

$$
\Delta \subset\left\{x \in R^{n}|| x \mid<q-T \eta\right\} .
$$

Fix any $0<\epsilon<T / 4$, and let $\Psi(t, x) \in C_{0}^{\infty}\left(R_{+}^{n+1}\right)$ such that

$$
\Psi(t, x)=1 \quad \text { for } \epsilon \leq t \leq T+\epsilon,|x| \leq 2 q .
$$

Since $\Psi v \in\left(\mathcal{D}^{\prime}\left(R_{+}^{n+1}\right)\right)^{n}$ has compact support, there is a positive integer $\nu$ such that

$$
\Psi v \in\left(H^{-\nu}\left(R_{+}^{n+1}\right)\right)^{n}
$$

and hence,

$$
\Psi v \in\left(H^{-\nu}\left(0, \infty ; H^{-\nu}\left(R^{n}\right)\right)\right)^{n} .
$$

In fact, we take $\nu \geq n$ to handle $B_{i j}\left(t, x_{1}, \ldots, x_{n} ; \omega\right)$. Next we choose a sequence of functions $\psi_{i}(t, x) \in C_{0}^{\infty}\left(R_{+}^{n+1}\right), i=1, \ldots, \nu+2$, such that $\Psi=1$ on the support of $\psi_{1}, \psi_{i}=1$ on the support of $\psi_{i+1}$, for $i=1, \ldots, \nu+1$, and $\psi_{\nu+2}=1$, for $2 \epsilon \leq t \leq T,|x| \leq q$. It is easy to see that

$$
\begin{aligned}
& \frac{\partial^{2}}{\partial t^{2}}\left(\psi_{1} v\right)-A\left(t, x, D_{x}\right)\left(\psi_{1} v\right) \\
= & \psi_{1} A\left(t, x, D_{x}\right) \int_{0}^{t} \frac{\partial^{n}}{\partial x_{1} \ldots \partial x_{n}} B\left(s, x_{1}, \ldots, x_{n} ; \omega\right) d s \\
& +(\text { a linear combination of } v \text { and first order derivatives of } v \\
& \left.\quad \text { which vanishes outside the support of } \psi_{1}\right),
\end{aligned}
$$

in the sense of distribution over $R_{+}^{n+1}$, where $B$ is $R^{n}$-valued, and its $i$ th component is $\sum_{j=1}^{n} B_{i j}\left(t, x_{1}, \ldots, x_{n} ; \omega\right)$.

Since $\psi_{1} v=\psi_{1} \Psi v \in\left(H^{-\nu}\left(0, \infty ; H^{-\nu}\left(R^{n}\right)\right)\right)^{n}$, it follows from (1.30)

$$
\psi_{1} v \in\left(H^{-\nu+1}\left(0, \infty ; H^{-\nu-2}\left(R^{n}\right)\right)\right)^{n} .
$$


By repetition of this procedure, we arrive at

$$
\psi_{\nu+2} v \in\left(H^{2}\left(0, \infty ; H^{-3 \nu-4}\left(R^{n}\right)\right)\right)^{n},
$$

and hence,

$$
\psi_{\nu+2} v \in\left(C\left((0, \infty) ; H^{-3 \nu-2}\left(R^{n}\right)\right)\right)^{n} \cap\left(C^{1}\left((0, \infty) ; H^{-3 \nu-4}\left(R^{n}\right)\right)\right)^{n} .
$$

By taking $t=T / 2$ as the initial time, we consider the forward and backward Cauchy problem

$$
\begin{aligned}
& L \theta=\Phi(x) A\left(t, x, D_{x}\right) \int_{0}^{t} \frac{\partial^{n}}{\partial x_{1} \ldots \partial x_{n}} B\left(s, x_{1}, \ldots, x_{n} ; \omega\right) d s, \\
& \theta(T / 2, x)=\left(\psi_{\nu+2} v\right)(T / 2, x), \quad \theta_{t}(T / 2, x)=\left(\psi_{\nu+2} v\right)_{t}(T / 2, x),
\end{aligned}
$$

where $\Phi(x) \in C_{0}^{\infty}\left(R^{n}\right)$ with $\Phi(x)=1$, for $|x| \leq q$.

By Theorem 1.1, there is a unique solution

$$
\theta \in\left(C\left([0, T] ; H^{-3 \nu-3}\left(R^{n}\right)\right)\right)^{n} \cap\left(C^{1}\left([0, T] ; H^{-3 \nu-4}\left(R^{n}\right)\right)\right)^{n} .
$$

But, by Corollary 1.3, (1.26), (1.30), (1.34), and (1.35), it holds that

$$
\psi(x) v(t, x)=\psi(x) \theta(t, x) \quad \text { in }[2 \epsilon, T] \times \Delta
$$

for every $\psi(x) \in C_{0}^{\infty}(\Delta)$. Now suppose we started out with a smaller number $\tilde{\epsilon}<\epsilon$. Let $\tilde{\nu}$ and $\tilde{\theta}$ correspond to $\tilde{\epsilon}$ in (1.28) through (1.37). Since $\theta(T / 2, x)=\tilde{\theta}(T / 2, x)$ and $\theta_{t}(T / 2, x)=\tilde{\theta}_{t}(T / 2, x)$ in the open ball $|x|<q$, it again follows from Corollary 1.3 and (1.26) that

$$
\psi(x) \theta(t, x)=\psi(x) \tilde{\theta}(t, x) \quad \text { in }[0, T] \times \Delta
$$

for all $\psi \in C_{0}^{\infty}(\Delta)$. Meanwhile, we have

$$
\psi(x) v(t, x)=\psi(x) \tilde{\theta}(t, x) \quad \text { in }[2 \tilde{\epsilon}, T] \times \Delta
$$

for all $\psi \in C_{0}^{\infty}(\Delta)$. By virtue of (1.37), (1.38), and (1.39), we can maintain the same $\nu$ for any smaller $\epsilon$ and conclude

$$
\psi(x) v(t, x)=\psi(x) \theta(t, x) \quad \text { in }(0, T] \times \Delta
$$

for every $\psi \in C_{0}^{\infty}(\Delta)$. Consequently, (1.23) and (1.24) follow with $m=3 \nu+4$.

LEMma 1.7. In the same setting as above, there is some $\Omega \in \mathcal{B}\left(\mathcal{S}^{\prime}\right)$ with $\mu(\Omega)=1$ such that for each $\omega \in \Omega, B_{i j}(0, x ; \omega)=0$ for all $x \in R^{n}$ and

$$
\begin{aligned}
& -\left\langle u_{t}(0, x ; \omega), \phi(0, x)\right\rangle_{\sharp}+\left\langle u(0, x ; \omega), \phi_{t}(0, x)\right\rangle_{\sharp} \\
& +\int_{0}^{\infty}\left\langle u(t, x ; \omega), L^{*} \phi(t, x)\right\rangle_{\sharp} d t \\
& =\sum_{i, j=1}^{n} \int_{R_{+}^{n+1}}(-1)^{n+1} \frac{\partial^{n+1} \phi_{i}(t, x)}{\partial t \cdots \partial x_{n}} B_{i j}\left(t, x_{1}, \ldots, x_{n} ; \omega\right) d t d x_{1} \cdots d x_{n}
\end{aligned}
$$


for every $\phi(t, x) \in\left(C_{0}^{\infty}\left(R^{n+1}\right)\right)^{n}$, where $\langle\cdot, \cdot\rangle_{\sharp}$ is the duality pairing between $\left(\mathcal{D}^{\prime}\left(R^{n}\right)\right)^{n}$ and $\left(C_{0}^{\infty}\left(R^{n}\right)\right)^{n}$.

Proof. By the same argument as in the first paragraph of the proof of Lemma 1.4, there is some $\Omega \in \mathcal{B}\left(\mathcal{S}^{\prime}\right)$ with $\mu(\Omega)=1$ such that for each $\omega \in \Omega, B_{i j}(0, x ; \omega)=0$, for all $x \in R^{n}$. By modifying $\Omega$, if necessary, we may assume that for each $\omega \in \Omega$, (1.21) holds for all $\phi \in\left(C_{0}^{\infty}\left(R^{n+1}\right)\right)^{n}$. Now let us fix $\omega \in \Omega$. Since the tensor product $C_{0}^{\infty}(R) \otimes C_{0}^{\infty}\left(R^{n}\right)$ is sequentially dense in $C_{0}^{\infty}\left(R^{n+1}\right)$, it is enough to consider $\phi$ in the form of $\alpha(t) \beta(x)$. Choose any $\alpha(t) \in C_{0}^{\infty}(R)$ and $\beta(x) \in\left(C_{0}^{\infty}\left(R^{n}\right)\right)^{n}$, and choose $T>0$ such that $\alpha(t)=0$ for $t \geq T$. We may take $\Delta$ and $q$ in (1.26) such that the support of $\beta \subset \Delta$. Since $B_{i j}(t, x ; \omega)$ is continuous in $(t, x) \in R^{n+1}$, we can extend the solution of (1.34) and (1.35) to a larger time interval so that

$$
\begin{gathered}
\langle u(t, x ; \omega), \beta(x)\rangle_{\sharp} \in C^{1}([-\epsilon, T+\epsilon]), \\
\left\langle u(t, x ; \omega), A^{*}\left(t, x, D_{x}\right) \beta(x)\right\rangle_{\sharp} \in C^{1}([-\epsilon, T+\epsilon])
\end{gathered}
$$

for some positive constant $\epsilon$, and

$$
\begin{aligned}
& \frac{d^{2}}{d t^{2}}\left\langle u * \rho_{h}, \beta\right\rangle_{\sharp}(t)-\left(\left\langle u, A^{*} \beta\right\rangle_{\sharp} * \rho_{h}\right)(t) \\
& =\frac{d}{d t} \sum_{i, j=1}^{n}\left\langle B_{i j} * \rho_{h},(-1)^{n} \frac{\partial^{n} \beta_{i}}{\partial x_{1} \cdots \partial x_{n}}\right\rangle_{\sharp}(t)
\end{aligned}
$$

for each $t \in(-\epsilon / 2, T+\epsilon / 2), 0<h<\epsilon / 2$, where $A^{*}\left(t, x, D_{x}\right)$ is the adjoint of $A\left(t, x, D_{x}\right), \quad \rho_{h}(t)=\rho(t / h) / h$, with $\rho(t) \in C_{0}^{\infty}(R)$ satisfying

$$
\rho(t)=\rho(-t) \geq 0 ; \quad \rho(t)=0 \text { for }|t| \geq 1 ; \quad \int_{-\infty}^{\infty} \rho(t) d t=1 .
$$

The convolution is taken with respect to the time variable.

Now we obtain (1.41) by going through the standard procedure: (i) multiply (1.44) by $\alpha(t)$ for $-\epsilon / 2<t<T+\epsilon / 2$; (ii) integrate over [0,T]; (iii) pass $h \rightarrow 0$ with the help of (1.42), (1.43), and the fact $B_{i j}(0, x ; \omega)=0$ for all $x \in R^{n}$.

Next we show that the traces of $u$ and $u_{t}$ with respect to the time variable can be defined as generalized stochastic processes.

Lemma 1.8. Let $u\left(t, x_{1}, \ldots, x_{n} ; \omega\right)$ be a solution of $(0.1)$ in $R_{+}^{n+1}$. Then, for each fixed $t_{0}>0, u\left(t_{0}, x_{1}, \ldots, x_{n} ; \omega\right)$ and $u_{t}\left(t_{0}, x_{1}, \ldots, x_{n} ; \omega\right)$ are generalized stochastic processes with respect to $\mathcal{F}\left(\mathcal{S}^{\prime}\right)$ which is the completion of $\mathcal{B}\left(\mathcal{S}^{\prime}\right)$.

Proof. Choose any $\beta\left(x_{1}, \ldots, x_{n}\right) \in\left(C_{0}^{\infty}\left(R^{n}\right)\right)^{n}$. We have to show that

$$
\left\langle u\left(t_{0}, x_{1}, \ldots, x_{n} ; \omega\right), \beta\left(x_{1}, \ldots, x_{n}\right)\right\rangle_{\sharp}
$$

and

$$
\left\langle u\left(t_{0}, x_{1}, \ldots, x_{n} ; \omega\right), \beta\left(x_{1}, \ldots, x_{n}\right)\right\rangle_{\sharp}
$$

are measurable with respect to $\mathcal{F}\left(\mathcal{S}^{\prime}\right)$. Here $\langle\cdot, \cdot\rangle_{\sharp}$ is the duality pairing between $\left(\mathcal{D}^{\prime}\left(R^{n}\right)\right)^{n}$ and $\left(C_{0}^{\infty}\left(R^{n}\right)\right)^{n}$. Choose a positive number $T$ and an open bounded subset $\Delta \subset R^{n}$ so that $t_{0}<T$ and the support of $\beta \subset \Delta$. Then, by Lemma 1.6, for each fixed $\omega \in \Omega$, where $\Omega \in \mathcal{B}\left(\mathcal{S}^{\prime}\right)$ with $\mu(\Omega)=1$,

$$
\left\langle u\left(t, x_{1}, \ldots, x_{n} ; \omega\right), \beta\left(x_{1}, \ldots, x_{n}\right)\right\rangle_{\sharp}
$$


is continuous in $t \in[0, T]$. Consequently,

$$
\begin{aligned}
& \left\langle u\left(t_{0}, x_{1}, \ldots, x_{n} ; \omega\right), \beta\left(x_{1}, \ldots, x_{n}\right)\right\rangle_{\sharp} \\
& \quad=\lim _{h \rightarrow 0} \ll u\left(t, x_{1}, \ldots, x_{n} ; \omega\right), \quad \rho_{h}\left(t-t_{0}\right) \beta\left(x_{1}, \ldots, x_{n}\right) \gg,
\end{aligned}
$$

where $\ll \cdot, \cdot \gg$ is the duality pairing between $\left(\mathcal{D}^{\prime}\left(R_{+}^{n+1}\right)\right)^{n}$ and $\left(C_{0}^{\infty}\left(R_{+}^{n+1}\right)\right)^{n}$, $\rho_{h}(t)=\rho(t / h) / h$ and $\rho(t)$ is the same as in (1.45). But, for each sufficiently small $h$,

$$
\ll u\left(t, x_{1}, \ldots, x_{n} ; \omega\right), \rho_{h}\left(t-t_{0}\right) \beta\left(x_{1}, \ldots, x_{n}\right) \gg
$$

is measurable with respect to $\mathcal{B}\left(\mathcal{S}^{\prime}\right)$, because $u$ is a generalized stochastic process with respect to $\mathcal{B}\left(\mathcal{S}^{\prime}\right)$. Since (1.46) holds for each $\omega \in \Omega$,

$$
\left\langle u\left(t_{0}, x_{1}, \ldots, x_{n} ; \omega\right), \beta\left(x_{1}, \ldots, x_{n}\right)\right\rangle_{\sharp}
$$

is measurable with respect to $\mathcal{F}\left(\mathcal{S}^{\prime}\right)$. By the same argument,

$$
\left\langle u_{t}\left(t_{0}, x_{1}, \ldots, x_{n} ; \omega\right), \beta\left(x_{1}, \ldots, x_{n}\right)\right\rangle_{\sharp}
$$

is also measurable with respect to $\mathcal{F}\left(\mathcal{S}^{\prime}\right)$.

By virtue of Theorem 1.1, Corollary 1.3, and Lemma 1.6, we have the uniqueness of the solution to (0.1) and (0.2) in the following form.

LEMMA 1.9. If $u_{1}$ and $u_{2}$ are solutions of (0.1) and (0.2), then

$$
u_{1}=u_{2} \quad \text { in } R_{+}^{n+1}
$$

for almost all $\omega$.

Let us write with the same $\eta$ as in Theorem 1.2,

$$
\Xi\left(t_{0}, x_{0} ; \eta\right)=\left\{(t, x) \in R^{n+1}\left|0 \leq t \leq t_{0},\right| x-x_{0} \mid \leq \eta\left(t_{0}-t\right)\right\},
$$

and let $d$ be the smallest integer larger than $(n / 2)-1$. Our main result is the following.

THEOREM 1.10. There is a continuous Gaussian process $V\left(t, x_{1}, \ldots, x_{n} ; \omega\right)$ with parameter $\left(t, x_{1}, \ldots, x_{n}\right) \in[0, \infty) \times R^{n}$ such that

(i) $L^{d} V$ is a unique solution of (0.1) and (0.2);

(ii) $V\left(t, x_{1}, \ldots, x_{n} ; \omega\right)$ and $V\left(\tilde{t}, \tilde{x}_{1}, \ldots, \tilde{x}_{n} ; \omega\right)$ are independent random variables if $\Xi\left(t, x_{1}, \ldots, x_{n} ; \eta\right)$ and $\Xi\left(\tilde{t}, \tilde{x}_{1}, \ldots, \tilde{x}_{n} ; \eta\right)$ are disjoint.

The operator $L$ was defined by (1.22).

2. Proof of the main result. The proof of Theorem 1.10 consists of two basic steps. First, we construct an integral kernel which represents a solution of the deterministic equation:

$$
L^{d} v=f \quad \text { in } R_{+}^{n+1}
$$

with zero initial conditions and $f \in L_{\text {loc }}^{2}\left([0, \infty) ;\left(L^{2}\left(R^{n}\right)\right)^{n}\right)$. For this, we use Theorems 1.1, 1.2, and Corollary 1.3. We then obtain approximate solutions by truncating the chaos expansion of the space-time white noise, and prove the convergence to the true solution. Approximation of the white noise is necessary to establish rigorously the existence of a solution according to Definition 1.5. 
2.1. Construction of an integral kernel. Consider the initial value problem

$$
\left\{\begin{array}{l}
L u^{(1)}=f \quad \text { in } R_{+}^{n+1}, \\
u^{(1)}(0, x)=0, u_{t}^{(1)}(0, x)=0 \quad \text { for } x \in R^{n},
\end{array}\right.
$$

where $f \in L_{l o c}^{2}\left([0, \infty) ;\left(L^{2}\left(R^{n}\right)\right)^{n}\right)$. Then there is a unique solution

$$
u^{(1)} \in C\left([0, \infty) ;\left(H^{1}\left(R^{n}\right)\right)^{n}\right) \cap C^{1}\left([0, \infty) ;\left(L^{2}\left(R^{n}\right)\right)^{n}\right),
$$

which satisfies

$$
\left\|u^{(1)}(t, \cdot)\right\|_{\left(H^{1}\left(R^{n}\right)\right)^{n}}+\left\|u_{t}^{(1)}(t, \cdot)\right\|_{\left(L^{2}\left(R^{n}\right)\right)^{n}} \leq C_{T}\|f\|_{L^{2}\left(0, T ;\left(L^{2}\left(R^{n}\right)\right)^{n}\right)}
$$

for all $0 \leq t \leq T$. Here and below, $C_{T}$ stands for generic positive constants depending only on $T>0$. Next we consider

$$
\left\{\begin{array}{l}
L u^{(2)}=u^{(1)} \quad \text { in } R_{+}^{n+1}, \\
u^{(2)}(0, x)=0, \quad u_{t}^{(2)}(0, x)=0 \quad \text { for } x \in R^{n} .
\end{array}\right.
$$

We have

$$
u^{(2)} \in C\left([0, \infty) ;\left(H^{2}\left(R^{n}\right)\right)^{n}\right) \cap C^{1}\left([0, \infty) ;\left(H^{1}\left(R^{n}\right)\right)^{n}\right),
$$

which satisfies

$$
\left\|u^{(2)}(t, \cdot)\right\|_{\left(H^{2}\left(R^{n}\right)\right)^{n}}+\left\|u_{t}^{(2)}(t, \cdot)\right\|_{\left(H^{1}\left(R^{n}\right)\right)^{n}} \leq C_{T}\|f\|_{L^{2}\left(0, T ;\left(L^{2}\left(R^{n}\right)\right)^{n}\right)}
$$

for all $0 \leq t \leq T$.

Inductively, we have for $i=1, \ldots, d$,

$$
\left\{\begin{array}{l}
L u^{(i+1)}=u^{(i)} \quad \text { in } R_{+}^{n+1}, \\
u^{(i+1)}(0, x)=0, u_{t}^{(i+1)}(0, x)=0 \quad \text { for } x \in R^{n} .
\end{array}\right.
$$

It follows that

$$
L^{d+1} u^{(d+1)}=f \quad \text { in } R_{+}^{n+1}
$$

and

$$
\begin{gathered}
u^{(d+1)} \in C\left([0, \infty) ;\left(H^{d+1}\left(R^{n}\right)\right)^{n}\right) \cap C^{1}\left([0, \infty) ;\left(H^{d}\left(R^{n}\right)\right)^{n}\right) ; \\
\left\|u^{(d+1)}(t, \cdot)\right\|_{\left(H^{d+1}\left(R^{n}\right)\right)^{n}}+\left\|u_{t}^{(d+1)}(t, \cdot)\right\|_{\left(H^{d}\left(R^{n}\right)\right)^{n}} \leq C_{T}\|f\|_{L^{2}\left(0, T ;\left(L^{2}\left(R^{n}\right)\right)^{n}\right)}
\end{gathered}
$$

for all $0 \leq t \leq T$. Recall that $d$ is the smallest integer larger than $(n / 2)-1$. Let $C_{B}\left(R^{n}\right)$ be the space of all uniformly bounded continuous functions on $R^{n}$. Since $H^{d+1}\left(R^{n}\right) \subset C_{B}\left(R^{n}\right)$, we find that for each fixed $0 \leq t_{0} \leq T, x_{0} \in R^{n}$, the mapping

$$
f \mapsto u^{(d+1)}\left(t_{0}, x_{0}\right)
$$

is a bounded linear mapping from $\left(L^{2}\left(0, T ; L^{2}\left(R^{n}\right)\right)\right)^{n}$ to $R^{n}$. Thus there is a matrix function $G=\left[G_{i j}\left(t, x ; t_{0}, x_{0}\right)\right]$ with each $G_{i j} \in L^{2}\left(0, T ; L^{2}\left(R^{n}\right)\right)$ so that

$$
u_{i}^{(d+1)}\left(t_{0}, x_{0}\right)=\int_{0}^{T} \int_{R^{n}} \sum_{j=1}^{n} G_{i j}\left(t, x ; t_{0}, x_{0}\right) f_{j}(t, x) d x d t .
$$


By Corollary 1.3, we find that modification of $f$ outside of $\cup_{0 \leq t \leq t_{0}}\left\{\left(t, \overline{\Gamma_{\left(t_{0}, x_{0}\right)}(t ; \epsilon, \eta)}\right)\right\}$ does not change the values of $u^{(k)}, k=1, \ldots, d+1$, in $\cup_{0 \leq t \leq t_{0}}\left\{\left(t, \overline{\Gamma_{\left(t_{0}, x_{0}\right)}(t ; \epsilon, \eta)}\right)\right\}$. Consequently, it holds that

$$
u_{i}^{(d+1)}\left(t_{0}, x_{0}\right)=\int_{0}^{t_{0}} \int_{\Gamma_{\left(t_{0}, x_{0}\right)}(t ; \epsilon, \eta)} \sum_{j=1}^{n} G_{i j}\left(t, x ; t_{0}, x_{0}\right) f_{j}(t, x) d x d t
$$

for every $\epsilon>0$. By passing $\epsilon \rightarrow 0$, we arrive at

$$
u_{i}^{(d+1)}\left(t_{0}, x_{0}\right)=\iint_{\Xi\left(t_{0}, x_{0} ; \eta\right)} \sum_{j=1}^{n} G_{i j}\left(t, x ; t_{0}, x_{0}\right) f_{j}(t, x) d x d t
$$

which, together with (2.11), implies

$$
\text { the support of } G_{i j}\left(t, x ; t_{0}, x_{0}\right) \subset \Xi\left(t_{0}, x_{0} ; \eta\right) \text {. }
$$

Lemma 2.1. For each $T>0$, the mapping $\left(t_{0}, x_{0}\right) \mapsto G_{i j}\left(\cdot ; t_{0}, x_{0}\right)$ is Hölder continuous from $[0, T] \times R^{n}$ to $L^{2}\left([0, T] \times R^{n}\right), i, j=1, \ldots, n$.

Proof. Let us write $d+1=(n / 2)+\epsilon$, where $\epsilon=1 / 2$ if $n$ is odd, and $\epsilon=1$ if $n$ is even. It follows from (2.8) and (2.9)

$$
\begin{aligned}
& \left|u^{(d+1)}\left(t_{0}, x_{0}\right)-u^{(d+1)}\left(\tilde{t}_{0}, x_{0}\right)\right| \\
\leq & C\left\|u^{(d+1)}\left(t_{0}, \cdot\right)-u^{(d+1)}\left(\tilde{t}_{0}, \cdot\right)\right\|_{\left(H^{(n / 2)+(\epsilon / 2)}\left(R^{n}\right)\right)^{n}} \\
\leq & C\left\|u^{(d+1)}\left(t_{0}, \cdot\right)-u^{(d+1)}\left(\tilde{t}_{0}, \cdot\right)\right\|_{\left(H^{(n / 2)+\epsilon}\left(R^{n}\right)\right)^{n}}^{1-(\epsilon / 2)}\left\|\int_{\tilde{t}_{0}}^{t_{0}} u_{t}^{(d+1)}(t, \cdot) d t\right\|_{\left(H^{(n / 2)+\epsilon-1}\left(R^{n}\right)\right)^{n}}^{\epsilon / 2} \\
\leq & C_{T}\left|t_{0}-\tilde{t}_{0}\right|^{\epsilon / 2}\|f\|_{L^{2}\left(0, T ;\left(L^{2}\left(R^{n}\right)\right)^{n}\right)}
\end{aligned}
$$

for all $t_{0}, \tilde{t}_{0} \in[0, T]$ and all $x_{0} \in R^{n}$. In the meantime, by (2.9) and the Sobolev imbedding theorem,

$$
\left|u^{(d+1)}\left(t_{0}, x_{0}\right)-u^{(d+1)}\left(t_{0}, \tilde{x}_{0}\right)\right| \leq C_{T}\left|x_{0}-\tilde{x}_{0}\right|^{\epsilon}\|f\|_{L^{2}\left(0, T ;\left(L^{2}\left(R^{n}\right)\right)^{n}\right)}
$$

for all $t_{0} \in[0, T]$ and all $x_{0}, \tilde{x}_{0} \in R^{n}$.

This, together with (2.11) and (2.15), yields

$$
\begin{aligned}
& \left|\int_{0}^{T} \int_{R^{n}} \sum_{j=1}^{n}\left(G_{i j}\left(t, x ; t_{0}, x_{0}\right)-G_{i j}\left(t, x ; \tilde{t}_{0}, \tilde{x}_{0}\right)\right) f_{j}(t, x) d x d t\right| \\
& \quad \leq C_{T}\left(\left|t_{0}-\tilde{t}_{0}\right|^{\epsilon / 2}+\left|x_{0}-\tilde{x}_{0}\right|^{\epsilon}\right)\|f\|_{\left(L^{2}\left([0, T] \times R^{n}\right)\right)^{n}}
\end{aligned}
$$

and thus, for each $i, j=1, \ldots, n$,

$$
\left\|G_{i j}\left(\cdot ; t_{0}, x_{0}\right)-G_{i j}\left(\cdot ; \tilde{t}_{0}, \tilde{x}_{0}\right)\right\|_{L^{2}\left([0, T] \times R^{n}\right)} \leq C_{T}\left(\left|t_{0}-\tilde{t}_{0}\right|^{\epsilon / 2}+\left|x_{0}-\tilde{x}_{0}\right|^{\epsilon}\right)
$$

for all $\left(t_{0}, x_{0}\right),\left(\tilde{t}_{0}, \tilde{x}_{0}\right) \in[0, T] \times R^{n}$. This proves the Hölder continuity of $G_{i j}$. 
2.2. Approximation and convergence. We approximate $F(t, x)$ in $(0.1)$ by

$$
F^{N}(t, x)=\left\{\begin{array}{l}
F(t, x) \quad \text { for }|t|+|x| \leq N \\
0 \quad \text { otherwise }
\end{array}\right.
$$

Recalling (1.20), we define

$$
Q_{i}^{N}(t, x ; \omega)=\sum_{k=1}^{N} \sum_{j=1}^{n} F_{i j}^{N}(t, x) \eta_{k}(t, x)\left\langle\omega_{j}, \eta_{k}\right\rangle .
$$

Then, for each $\omega$,

$$
Q_{i}^{N}(t, x ; \omega) \in L^{2}\left(R_{+}^{n+1}\right), \quad i=1, \ldots, n
$$

Next we define

$$
W_{i}^{N}(t, x ; \omega)=\int_{0}^{\infty} \int_{R^{n}} \sum_{j=1}^{n} G_{i j}(s, y ; t, x) Q_{j}^{N}(s, y ; \omega) d y d s .
$$

Let $W^{N}=\left(W_{1}^{N}, \ldots, W_{n}^{N}\right)$, and $Q^{N}=\left(Q_{1}^{N}, \ldots, Q_{n}^{N}\right)$. Then it holds that for each $\omega$,

$$
L^{d+1} W^{N}=Q^{N}(t, x ; \omega) \quad \text { in } R_{+}^{n+1},
$$

and, for $j=0,1, \ldots, d$,

$$
\begin{gathered}
L^{j} W^{N} \in C\left([0, \infty) ;\left(H^{d+1-j}\left(R^{n}\right)\right)^{n}\right) \cap C^{1}\left([0, \infty) ;\left(H^{d-j}\left(R^{n}\right)\right)^{n}\right), \\
\left(L^{j} W^{N}\right)(0, x ; \omega)=0, \quad\left(L^{j} W^{N}\right)_{t}(0, x ; \omega)=0 \quad \text { in } R^{n} .
\end{gathered}
$$

Choose any $\mathcal{M} \in \mathcal{B}\left(\mathcal{S}^{\prime}\right)$. By virtue of the special structure of $W_{i}^{N}$ defined by $(2.20)$ and (2.22), it is apparent that

$$
\iint_{R_{+}^{n+1}} \int_{\mathcal{M}} W^{N} \cdot\left(\left(L^{*}\right)^{d+1} \phi\right) d \mu d x d t=\int_{\mathcal{M}} \iint_{R_{+}^{n+1}} W^{N} \cdot\left(\left(L^{*}\right)^{d+1} \phi\right) d x d t d \mu
$$

for all $\phi \in\left(C_{0}^{\infty}\left(R^{n+1}\right)\right)^{n}$. At the same time, we use (2.21), (2.23), (2.24), (2.25), and the same argument as in the proof of Lemma 1.7 to find that

$$
\int_{\mathcal{M}} \iint_{R_{+}^{n+1}} W^{N} \cdot\left(\left(L^{*}\right)^{d+1} \phi\right) d x d t d \mu=\iint_{\mathcal{M}_{+}^{n+1}} \iint_{R^{n}} Q^{N} \cdot \phi d x d t d \mu
$$

for all $\phi \in\left(C_{0}^{\infty}\left(R^{n+1}\right)\right)^{n}$.

Recalling (1.20), (2.20), and (2.22), we define, for $i=1, \ldots, n$,

$$
W_{i}(t, x ; \omega)=\sum_{\nu, j=1}^{n}\left\langle\omega_{j}(s, y), G_{i \nu}(s, y ; t, x) F_{\nu j}(s, y)\right\rangle,
$$


and write $W=\left(W_{1}, \ldots, W_{n}\right)$. We can use (1.7) in the same way as for (1.20) to find that for each $g \in\left(L^{2}\left(R^{n+1}\right)\right)^{n}$,

$$
\sum_{k=1}^{N} \sum_{j=1}^{n}\left\langle\omega_{j}(z), \eta_{k}(z)\right\rangle \int_{R^{n+1}} \eta_{k}(z) g_{j}(z) d z \rightarrow \sum_{j=1}^{n}\left\langle\omega_{j}(z), g_{j}(z)\right\rangle,
$$

in $L^{2}\left(\mathcal{S}^{\prime}, d \mu\right)$. The assumption $(\mathrm{V}),(2.14),(2.18)$, and (2.29) imply that as $N \rightarrow \infty$

$$
W_{i}^{N}(t, x ; \omega) \rightarrow W_{i}(t, x ; \omega) \quad \text { in } L^{2}\left(\mathcal{S}^{\prime}, d \mu\right),
$$

uniformly in $(t, x)$ of each bounded subset of $[0, \infty) \times R^{n}$. Hence, we find that for every $\phi \in\left(C_{0}^{\infty}\left(R^{n+1}\right)\right)^{n}$,

$$
\iint_{R_{+}^{n+1}} \int_{\mathcal{M}} W^{N} \cdot\left(\left(L^{*}\right)^{d+1} \phi\right) d \mu d x d t \rightarrow \iint_{R_{+}^{n+1}} \int_{\mathcal{M}} W \cdot\left(\left(L^{*}\right)^{d+1} \phi\right) d \mu d x d t
$$

as $N \rightarrow \infty$.

Meanwhile, for every $\phi \in\left(C_{0}^{\infty}\left(R^{n+1}\right)\right)^{n}$, as $N \rightarrow \infty$,

$$
\int_{\mathcal{M}} \iint_{R_{+}^{n+1}} Q^{N} \cdot \phi d x d t d \mu \rightarrow \int_{\mathcal{M}} \sum_{i, j=1}^{n}\left\langle\omega_{j}(t, x), F_{i j}(t, x) \phi_{i}(t, x) \pi_{+}(t)\right\rangle d \mu,
$$

where

$$
\pi_{+}(t)= \begin{cases}1 & \text { for } t \geq 0 \\ 0 & \text { otherwise. }\end{cases}
$$

Thus, for every $\phi \in\left(C_{0}^{\infty}\left(R^{n+1}\right)\right)^{n}$,

$$
\iint_{R_{+}^{n+1}} \int_{\mathcal{M}} W \cdot\left(\left(L^{*}\right)^{d+1} \phi\right) d \mu d x d t=\int_{\mathcal{M}} \sum_{i, j=1}^{n}\left\langle\omega_{j}, F_{i j} \phi_{i} \pi_{+}\right\rangle d \mu .
$$

Next we will obtain a continuous version of $W$. Let $K$ be a bounded subset of $[0, \infty) \times$ $R^{n}$. It follows from (1.7) and (2.18) that for each positive integer $m$,

$$
E\left(\left|W_{i}(t, x ; \omega)-W_{i}(\tilde{t}, \tilde{x} ; \omega)\right|^{2 m}\right) \leq C_{m, K}|(t, x)-(\tilde{t}, \tilde{x})|^{\epsilon m}
$$

for all $(t, x),(\tilde{t}, \tilde{x}) \in K$, for some positive constant $C_{m, K}$. By a partition of unity and Kolmogorov's theorem, there is a continuous version $V_{i}(t, x ; \omega)$ of $W_{i}(t, x ; \omega)$. We write $V=\left(V_{1}, \ldots, V_{n}\right)$. It is evident that $V$ is a continuous Gaussian process with parameter $(t, x) \in[0, \infty) \times R^{n}$.

Lemma 2.2. For every $\phi \in\left(C_{0}^{\infty}\left(R^{n+1}\right)\right)^{n}$, and every $\mathcal{M} \in \mathcal{B}\left(\mathcal{S}^{\prime}\right)$, it holds that

$$
\begin{aligned}
& \int_{\mathcal{M}} \iint_{R_{+}^{n+1}} V \cdot\left(\left(L^{*}\right)^{d+1} \phi\right) d x d t d \mu \\
& =\int_{\mathcal{M}} \sum_{i, j=1}^{n} \iint_{R_{+}^{n+1}}(-1)^{n+1} \frac{\partial^{n+1} \phi_{i}}{\partial t \partial x_{1} \cdots \partial x_{n}} B_{i j}(t, x ; \omega) d x d t d \mu .
\end{aligned}
$$


Proof. Since $E\left(\left|W_{i}(t, x ; \cdot)\right|^{2}\right)$ is bounded uniformly in $(t, x)$ of each bounded subset of $[0, \infty) \times R^{n}$, it is apparent that

$$
\iint_{R_{+}^{n+1}} \int_{\mathcal{M}}\left|W \cdot\left(\left(L^{*}\right)^{d+1} \phi\right)\right| d \mu d x d t<\infty
$$

for each $\phi \in\left(C_{0}^{\infty}\left(R^{n+1}\right)\right)^{n}$. In the meantime, $V_{i}(t, x ; \omega)=W_{i}(t, x ; \omega)$ for almost all $\omega$, for each fixed $(t, x)$ so that

$$
\iint_{R_{+}^{n+1}} \int_{\mathcal{M}} W \cdot\left(\left(L^{*}\right)^{d+1} \phi\right) d \mu d x d t=\iint_{R_{+}^{n+1}} \int_{\mathcal{M}} V \cdot\left(\left(L^{*}\right)^{d+1} \phi\right) d \mu d x d t
$$

and also,

$$
\iint_{R_{+}^{n+1}} \int_{\mathcal{M}}\left|W \cdot\left(\left(L^{*}\right)^{d+1} \phi\right)\right| d \mu d x d t=\iint_{R_{+}^{n+1}} \int_{\mathcal{M}}\left|V \cdot\left(\left(L^{*}\right)^{d+1} \phi\right)\right| d \mu d x d t
$$

for every $\phi \in\left(C_{0}^{\infty}\left(R^{n+1}\right)\right)^{n}$. Furthermore, $V_{i}(t, x ; \omega)$ is continuous in $(t, x)$ for each $\omega$, and measurable with respect to $\mathcal{B}\left(\mathcal{S}^{\prime}\right)$ for each $(t, x)$. Hence, $V_{i}$ is measurable with respect to the product $\sigma$-algebra. By Fubini's theorem, we have

$$
\iint_{R_{+}^{n+1}} \int_{\mathcal{M}} V \cdot\left(\left(L^{*}\right)^{d+1} \phi\right) d \mu d x d t=\int_{\mathcal{M}_{R_{+}^{n+1}}} \iint_{V} V \cdot\left(\left(L^{*}\right)^{d+1} \phi\right) d x d t d \mu .
$$

Meanwhile, it follows from Lemma 1.4 that for every $\phi \in\left(C_{0}^{\infty}\left(R^{n+1}\right)\right)^{n}$,

$$
\begin{aligned}
& \int_{\mathcal{M}} \sum_{i, j=1}^{n}<\omega_{j}, F_{i j} \phi_{i}>d \mu \\
& =\int_{\mathcal{M}} \sum_{i, j=1}^{n} \iint_{R^{n+1}}(-1)^{n+1} \frac{\partial^{n+1} \phi_{i}}{\partial t \partial x_{1} \ldots \partial x_{n}} B_{i j}\left(t, x_{1}, \ldots, x_{n} ; \omega\right) d x d t d \mu .
\end{aligned}
$$

Let us choose $\pi(t) \in C^{\infty}(R)$ with $\pi(t)=1$ for $t \geq 0$, and $\pi(t)=0$ for $t \leq-1$, and write

$$
\pi^{h}(t)=\pi(t / h) \quad \text { for } h>0 .
$$

Then (2.41) is valid with $\phi_{i}$ replaced by $\phi_{i} \pi^{h}$, and it is easy to see that as $h \rightarrow 0$,

$$
\sum_{i, j=1}^{n}\left\langle\omega_{j}, F_{i j} \phi_{i} \pi^{h}\right\rangle \rightarrow \sum_{i, j=1}^{n}\left\langle\omega_{j}, F_{i j} \phi_{i} \pi_{+}\right\rangle \quad \text { in } L^{2}\left(\mathcal{S}^{\prime}, d \mu\right) .
$$

Since $B_{i j}(t, x ; \omega)$ is continuous in $(t, x)$ for each $\omega$, it follows from (1.15) and Fatou's lemma that for each bounded subset $K \subset[0, \infty) \times R^{n}$,

$$
E\left(\left|B_{i j}(t, x ; \cdot)\right|^{2}\right) \leq C_{K}
$$


for all $(t, x) \in K$, for some positive constant $C_{K}$. We use this for the following procedure:

$$
\begin{aligned}
& \lim _{h \rightarrow 0} \int_{\mathcal{M}} \iint_{R^{n+1}} \frac{\partial^{n} \phi_{i}(t, x)}{\partial x_{1} \cdots \partial x_{n}} \frac{1}{h} \pi^{\prime}(t / h) B_{i j}(t, x ; \omega) d x d t d \mu \\
& =\lim _{h \rightarrow 0} \int_{-1}^{0} \int_{|x| \leq q} \int_{\mathcal{M}} \frac{\partial^{n} \phi_{i}}{\partial x_{1} \cdots \partial x_{n}}(h s, x) \pi^{\prime}(s) B_{i j}(h s, x ; \omega) d \mu d x d s \\
& =\int_{-1}^{0} \int_{|x| \leq q}\left(\lim _{h \rightarrow 0} \int_{\mathcal{M}} \frac{\partial^{n} \phi_{i}}{\partial x_{1} \cdots \partial x_{n}}(h s, x) \pi^{\prime}(s) B_{i j}(h s, x ; \omega) d \mu\right) d x d s \\
& =0 .
\end{aligned}
$$

Here the second equality is justified by the fact that

$$
\int_{\mathcal{M}} \frac{\partial^{n} \phi_{i}}{\partial x_{1} \cdots \partial x_{n}}(h s, x) \pi^{\prime}(s) B_{i j}(h s, x ; \omega) d \mu
$$

is bounded uniformly in $0 \leq h \leq 1,-1 \leq s \leq 0$, and $|x| \leq q$, where $q$ is a positive number such that $\phi_{i}(t, x)=0$ for $|x| \geq q$. For the last equality, we argue as follows. First, for each fixed $s$ and $x$, as $h \rightarrow 0$,

$$
<\omega_{j}(y), F_{i j}(y) \chi_{\left(h s, x_{1}, \ldots, x_{n}\right)}(y)>\rightarrow 0 \quad \text { in } L^{2}\left(\mathcal{S}^{\prime}, d \mu\right) .
$$

Next, since $B_{i j}(t, x ; \omega)$ is a continuous version of the process defined by (1.10), we have for each fixed $s$ and $x$,

$$
\begin{aligned}
\lim _{h \rightarrow 0} \int_{\mathcal{M}} B_{i j}(h s, x ; \omega) d \mu & =\lim _{h \rightarrow 0} \int_{\mathcal{M}}<\omega_{j}(y), F_{i j}(y) \chi_{\left(h s, x_{1}, \ldots, x_{n}\right)}(y)>d \mu \\
& =0 .
\end{aligned}
$$

Hence, the last equality follows.

Similarly, we also have

$$
\begin{aligned}
& \lim _{h \rightarrow 0} \int_{\mathcal{M}} \iint_{R^{n+1}} \frac{\partial^{n+1} \phi_{i}(t, x)}{\partial t \cdots \partial x_{n}} \pi^{h}(t, x) B_{i j}(t, x ; \omega) d x d t d \mu \\
& =\int_{\mathcal{M}} \iint_{R_{+}^{n+1}} \frac{\partial^{n+1} \phi_{i}(t, x)}{\partial t \cdots \partial x_{n}} B_{i j}(t, x ; \omega) d x d t d \mu .
\end{aligned}
$$

Combining (2.43), (2.45), and (2.48), we finally arrive at

$$
\begin{aligned}
& \int_{\mathcal{M}} \sum_{i, j=1}^{n}\left\langle\omega_{j}, F_{i j} \phi_{i} \pi_{+}\right\rangle d \mu \\
& =\int_{\mathcal{M}} \sum_{i, j=1}^{n} \iint_{R_{+}^{n+1}}(-1)^{n+1} \frac{\partial^{n+1} \phi_{i}}{\partial t \partial x_{1} \cdots \partial x_{n}} B_{i j}(t, x ; \omega) d x d t d \mu
\end{aligned}
$$

for all $\phi \in\left(C_{0}^{\infty}\left(R^{n+1}\right)\right)^{n}$. By means of (2.34), (2.38), (2.40), and (2.49), we derive (2.36) and conclude the proof of Lemma 2.2. 
We now show that $L^{d} V(t, x ; \omega)$ is a solution of $(0.1)$ according to Definition 1.5. (2.36) implies that for each $\phi \in\left(C_{0}^{\infty}\left(R_{+}^{n+1}\right)\right)^{n}$,

$$
\ll V,\left(L^{*}\right)^{d+1} \phi \gg=\sum_{i, j=1}^{n} \iint_{R_{+}^{n+1}}(-1)^{n+1} \frac{\partial^{n+1} \phi_{i}}{\partial t \cdots \partial x_{n}} B_{i j}(t, x ; \omega) d x d t
$$

holds for almost all $\omega$. Let $K$ be a compact subset of $R_{+}^{n+1}$. Then $C_{0}^{\infty}(K)$ is a separable Fréchet space. Hence, there is a countable dense subset $\left\{\phi^{(\nu)}\right\}_{\nu=1}^{\infty} \subset\left(C_{0}^{\infty}(K)\right)^{n}$. For each $\phi^{(\nu)}$, there is $\Omega_{\nu} \in \mathcal{B}\left(\mathcal{S}^{\prime}\right)$ such that $\mu\left(\Omega_{\nu}\right)=1$, and, for all $\omega \in \Omega_{\nu}$,

$$
\ll V, \quad\left(L^{*}\right)^{d+1} \phi^{(\nu)} \gg=\sum_{i, j=1}^{n} \iint_{R_{+}^{n+1}}(-1)^{n+1} \frac{\partial^{n+1} \phi_{i}^{(\nu)}}{\partial t \cdots \partial x_{n}} B_{i j}(t, x ; \omega) d x d t
$$

holds. Let $\Omega_{K}=\cap_{\nu=1}^{\infty} \Omega_{\nu}$. Then, $\mu\left(\Omega_{K}\right)=1$, and for all $\omega \in \Omega_{K}$, (2.51) holds for every $\phi \in\left(C_{0}^{\infty}(K)\right)^{n}$. Since $R_{+}^{n+1}$ is a countable union of compact subsets, there is $\Omega \in \mathcal{B}\left(\mathcal{S}^{\prime}\right)$ such that $\mu(\Omega)=1$, and for every $\omega \in \Omega$, (2.50) holds for all $\phi \in\left(C_{0}^{\infty}\left(R_{+}^{n+1}\right)\right)^{n}$. Hence, $L^{d} V$ is a solution of (0.1) in $R_{+}^{n+1}$.

Next we will show that $L^{d} V$ satisfies the initial conditions (0.2). Choose any $\gamma_{1}(x), \gamma_{2}(x) \in\left(C_{0}^{\infty}\left(R^{n}\right)\right)^{n}$, and consider the Cauchy problem:

$$
\begin{gathered}
L^{*} \psi=0 \quad \text { in }(-\infty, \infty) \times R^{n}, \\
\psi(0, x)=\gamma_{1}(x), \quad \psi_{t}(0, x)=\gamma_{2}(x) \quad \text { in } R^{n} .
\end{gathered}
$$

Choose a function $\zeta(t) \in C_{0}^{\infty}(R)$ such that $\zeta(t)=1$ for $|t| \leq 1$, and $\zeta(t)=0$ for $|t| \geq$ 2. Let us set $\sigma(t, x)=\zeta(t) \psi(t, x)$. The solution of the above Cauchy problem satisfies the property of a domain of dependence, and consequently, $\sigma(t, x) \in\left(C_{0}^{\infty}\left(R^{n+1}\right)\right)^{n}$. Furthermore, it is easy to see

$$
L^{*} \sigma=2 \zeta_{t} \psi_{t}+\zeta_{t t} \psi \in\left(C_{0}^{\infty}\left(R^{n+1}\right)\right)^{n},
$$

where the right-hand side vanishes for $|t| \leq 1$. In the meantime, by the same argument as above, we can infer from (2.36) that there is some $\Omega \in \mathcal{B}\left(\mathcal{S}^{\prime}\right)$ with $\mu(\Omega)=1$ such that for each $\omega \in \Omega$,

$$
\iint_{R_{+}^{n+1}} V \cdot\left(\left(L^{*}\right)^{d+1} \phi\right) d x d t=\sum_{i, j=1}^{n} \iint_{R_{+}^{n+1}}(-1)^{n+1} \frac{\partial^{n+1} \phi_{i}}{\partial t \cdots \partial x_{n}} B_{i j}(t, x ; \omega) d x d t
$$

holds for all $\phi \in\left(C_{0}^{\infty}\left(R^{n+1}\right)\right)^{n}$. With $\phi=\sigma$, the left-hand side can be written as

$$
\iint_{R_{+}^{n+1}} V \cdot\left(\left(L^{*}\right)^{d} L^{*} \sigma\right) d x d t=\int_{0}^{\infty}\left\langle L^{d} V, L^{*} \sigma\right\rangle_{\sharp} d t,
$$

because $L^{*} \sigma \in\left(C_{0}^{\infty}\left(R_{+}^{n+1}\right)\right)^{n}$ for $t \geq 0$. Thus (2.55) yields

$$
\begin{aligned}
\int_{0}^{\infty} & \left\langle L^{d} V(t, x ; \omega), L^{*} \sigma(t, x)\right\rangle_{\sharp} d t \\
= & \sum_{i, j=1}^{n} \iint_{R_{+}^{n+1}}(-1)^{n+1} \frac{\partial^{n+1} \sigma_{i}(t, x)}{\partial t \cdots \partial x_{n}} B_{i j}(t, x ; \omega) d x d t
\end{aligned}
$$


for all $\omega \in \Omega$. On the other hand, Lemma 1.7 implies that

$$
\begin{aligned}
& -\left\langle\left(L^{d} V\right)_{t}(0, x ; \omega), \gamma_{1}(x)\right\rangle_{\sharp}+\left\langle L^{d} V(0, x ; \omega), \gamma_{2}(x)\right\rangle_{\sharp} \\
& +\int_{0}^{\infty}\left\langle L^{d} V(t, x ; \omega), L^{*} \sigma(t, x)\right\rangle_{\sharp} d t \\
& =\sum_{i, j=1}^{n} \iint_{R_{+}^{n+1}}(-1)^{n+1} \frac{\partial^{n+1} \sigma_{i}(t, x)}{\partial t \cdots \partial x_{n}} B_{i j}(t, x ; \omega) d x d t
\end{aligned}
$$

for all $\omega \in \Omega$, after modification of $\Omega$ if necessary. But we note that this $\Omega$ is independent of $\gamma_{1}$ and $\gamma_{2}$. By comparing (2.57) and (2.58), we conclude that

$$
L^{d} V(0, x ; \omega)=0, \quad\left(L^{d} V\right)_{t}(0, x ; \omega)=0 \quad \text { in } R^{n}
$$

for each $\omega \in \Omega$, because $\gamma_{1}$ and $\gamma_{2}$ were chosen arbitrarily. The uniqueness is given by Lemma 1.9

Now the statement (i) of Theorem 1.10 has been established. The statement (ii) follows easily from (1.6), (2.14), and (2.28).

Final remark. Our goal is to obtain a solution in the form (0.6). If one is interested only in the existence of weak solutions for almost all $\omega$, there is a somewhat direct approach. It follows from Lemma 1.4 that $F \xi \in\left(H_{l o c}^{-1}\left(R ; H_{l o c}^{-n}\left(R^{n}\right)\right)\right)^{n}$ for almost all $\omega$. Since Theorem 1.1 cannot be applied directly for this function class, it requires some work to obtain a weak solution. This involves localization in the space variables and handling low regularity in the time variable as in the proof of Lemma 1.6. It also needs some extra work to show that the resulting weak solution is a generalized stochastic process. Meanwhile, the structure of such a solution is intractable. The reward for our special procedure for the existence of solution is twofold. First, the representation formula shows globally uniform structure of the solution as a generalized stochastic process. Second, our procedure shows that the solution can be approximated by a sequence of ordinary stochastic processes.

\section{REFERENCES}

[1] E.M. CABAÑA, The vibrating string forced by white noise, Z. Wahr scheinlich Keits theorie und Verw, Gebiete, 15 (1970), pp. 111-130.

[2] R. Courant and D. Hilbert, Methods of Mathematical Physics, Vol. 2, Interscience Publishers, New York, London, Sydney, 1962.

[3] G. Da Prato and J. Zabczyk, Stochastic Equations in Infinite Dimensions, Cambridge University Press, Cambridge, UK, 1992.

[4] R.C. Dalang And N.E. Frangos, The stochastic wave equation in two spatial dimensions, Ann. Probab., 26 (1998), pp. 187-212.

[5] G.F.D. DufF, The Cauchy problem for elastic waves in an anisotropic medium, Philos. Trans. Roy. Soc. London Ser. A, 252 (1960), pp. 249-273.

[6] B. Gaveau, The Cauchy problem for the stochastic wave equation, Bull. Sci. Math., 119 (1995), pp. 381-407.

[7] H. Holden, B. Øksendal, J. Ubøe, T. Zhang, Stochastic Partial Differential Equations: A Modeling, White Noise Functional Approach, Birkhäuser, Boston, 1996.

[8] I. Karatzas and S.E. Shreve, Brownian Motion and Stochastic Calculus, 2nd ed., SpringerVerlag, New York, 1991.

[9] J.U. KIM, On the local regularity of solutions in linear viscoelasticity of several space dimensions, Trans. Amer. Math. Soc., 346 (1994), pp. 359-398.

[10] M. Marcus And V. Mizel, Stochastic hyperbolic systems and the wave equation, Stochastics and Stochastics Reports, 36 (1991), pp. 225-244.

[11] C. Mueller, Long time existence for the wave equation with a noise term, Ann. Probab., 25 (1997), pp. 133-151. 
[12] D. Nualart, The Malliavin Calculus and Related Topics, Springer-Verlag, New York, 1995.

[13] E. Orsingher, Damped vibrations excited by white noise, Adv. in Appl. Probab., 16 (1984), pp. $562-584$.

[14] M. Riesz, L'intégrale de Riemann-Liouville et le problème de Cauchy, Acta Math., 81 (1949), pp. $1-223$.

[15] J.B. WALSH, An Introduction to Stochastic Partial Differential Equations, Lecture Notes in Math. 1180, Springer, Berlin, New York, 1986. 